\title{
A reverse Mulholland-type inequality in the whole plane
}

\author{
Jianquan Liao* (10) and Bicheng Yang
}

"Correspondence: lamth@163.com Department of Mathematics, Guangdong University of Education, Guangzhou, P.R. China

\section{Abstract \\ We present a new reverse Mulholland-type inequality in the whole plane with a best possible constant factor by introducing multiparameters, applying weight coefficients, and using the Hermite-Hadamard inequality. Moreover, we consider equivalent forms and some particular cases.}

MSC: 26D15; 47A07

Keywords: Mulholland-type inequality; Parameter; Weight coefficient; Equivalent form; Reverse

\section{Introduction}

Assuming that $p>1, \frac{1}{p}+\frac{1}{q}=1, a_{m}, b_{n} \geq 0,0<\sum_{m=1}^{\infty} a_{m}^{p}<\infty$, and $0<\sum_{n=1}^{\infty} b_{n}^{q}<\infty$, the Hardy-Hilbert inequality is provided as follows (see [1]):

$$
\sum_{n=1}^{\infty} \sum_{m=1}^{\infty} \frac{a_{m} b_{n}}{m+n}<\frac{\pi}{\sin (\pi / p)}\left(\sum_{m=1}^{\infty} a_{m}^{p}\right)^{\frac{1}{p}}\left(\sum_{n=1}^{\infty} b_{n}^{q}\right)^{\frac{1}{q}},
$$

where $\frac{\pi}{\sin (\pi / p)}$ is the best possible constant factor. By Theorem 343 in [1] (replacing $\frac{a_{m}}{m}$ and $\frac{b_{n}}{n}$ by $a_{m}$ and $b_{n}$, respectively), it yields the following Mulholland inequality with the same best value:

$$
\sum_{n=2}^{\infty} \sum_{m=2}^{\infty} \frac{a_{m} b_{n}}{\ln m n}<\frac{\pi}{\sin (\pi / p)}\left(\sum_{m=2}^{\infty} \frac{a_{m}^{p}}{m}\right)^{\frac{1}{p}}\left(\sum_{n=2}^{\infty} \frac{b_{n}^{q}}{n}\right)^{\frac{1}{q}} .
$$

Inequalities (1) and (2) play an important role in analysis and its applications (see [1, 2]). In 2007, Yang [3] published a Hilbert-type integral inequality in the whole plane. Various extensions of (1)-(2) and Yang's work have been presented since then [4-12]. Recently, Yang and Chen [13] presented the following extension of (1) in the whole plane:

$$
\begin{aligned}
& \sum_{|n|=1}^{\infty} \sum_{|m|=1}^{\infty} \frac{a_{m} b_{n}}{(|m-\xi|+|n-\eta|)^{\lambda}} \\
& \quad<2 B\left(\lambda_{1}, \lambda_{2}\right)\left[\sum_{|m|=1}^{\infty}|m-\xi|^{p\left(1-\lambda_{1}\right)-1} a_{m}^{p}\right]^{\frac{1}{p}}\left[\sum_{|n|=1}^{\infty}|n-\eta|^{q\left(1-\lambda_{2}\right)-1} b_{n}^{q}\right]^{\frac{1}{q}},
\end{aligned}
$$

(0) The Author(s) 2018. This article is distributed under the terms of the Creative Commons Attribution 4.0 International License (http://creativecommons.org/licenses/by/4.0/), which permits unrestricted use, distribution, and reproduction in any medium, provided you give appropriate credit to the original author(s) and the source, provide a link to the Creative Commons license, and indicate if changes were made. 
where the constant factor $2 B\left(\lambda_{1}, \lambda_{2}\right)\left(0<\lambda_{1}, \lambda_{2} \leq 1, \lambda_{1}+\lambda_{2}=\lambda, \xi, \eta \in\left[0, \frac{1}{2}\right]\right)$ is the best possible. In addition, Xin et al. [14] also carried out a similar result, and Zhong et al. [15] gave the reverse Mulholland's inequality in the whole plane.

In this paper, we present a new reverse Mulholland-type inequality in the whole plane with a best possible constant factor, which is similar to the results of [13], via introducing multiparameters, applying weight coefficients, and using the Hermite-Hadamard inequality. Moreover, we consider equivalent forms and some particular cases.

\section{An example and two lemmas}

We further assume that $\lambda_{1}, \lambda_{2}>0, \lambda_{1}+\lambda_{2}=\lambda \leq 1, \xi, \eta \in\left[0, \frac{1}{2}\right], \alpha, \beta \in\left[\arccos \frac{1}{3}, \frac{\pi}{2}\right]$, and

$$
k_{\gamma}\left(\lambda_{1}\right):=\frac{2 \pi^{2} \csc ^{2} \gamma}{\lambda^{2} \sin ^{2}\left(\frac{\pi \lambda_{1}}{\lambda}\right)} \quad(\gamma=\alpha, \beta)
$$

Remark 1 Since $\alpha, \beta \in\left[\arccos \frac{1}{3}, \frac{\pi}{2}\right], \xi, \eta \in\left[0, \frac{1}{2}\right]$, it follows that

$$
\left(\frac{3}{2} \pm \eta\right)(1 \mp \cos \beta) \geq 1 \quad \text { and } \quad\left(\frac{3}{2} \pm \xi\right)(1 \mp \cos \alpha) \geq 1
$$

Example 1 We set $g(u):=\frac{\ln u}{u-1}(u>0), g(1):=\lim _{u \rightarrow 1} g(u)=1$. Then we have $g(u)>0, g^{\prime}(u)<$ $0, g^{\prime \prime}(u)>0(u>0)$. By Tailor's formula we find

$$
g(u)=\frac{\ln [1+(u-1)]}{u-1}=\sum_{k=0}^{\infty}(-1)^{k} \frac{(u-1)^{k}}{k+1}=\sum_{k=0}^{\infty} \frac{(-1)^{k} k !}{k+1} \frac{(u-1)^{k}}{k !} \quad(-1<u-1 \leq 1),
$$

and then $g^{(k)}(1)=\frac{(-1)^{k} k !}{k+1}(k=0,1,2, \ldots)$. Hence, $g^{(0)}(1)=g(1)=1, g^{\prime}(1)=-\frac{1}{2}, g^{\prime \prime}(1)=\frac{2}{3}$. It is evident that $g(u)>0$. We obtain $g^{\prime}(u)=\frac{h(u)}{u(u-1)^{2}}, h(u):=u-1-u \ln u$. Since

$$
h^{\prime}(u)=-\ln u>0 \quad(0<u<1) ; \quad h^{\prime}(u)=-\ln u<0 \quad(u>1),
$$

it follows that $h_{\max }=h(1)=0$ and $h(u)<0(u \neq 1)$. Then we have $g^{\prime}(u)<0(u \neq 1)$. Since $g^{\prime}(1)=-\frac{1}{2}<0$, it follows that $g^{\prime}(u)<0(u>0)$. We find

$$
g^{\prime \prime}(u)=\frac{J(u)}{u^{2}(u-1)^{3}}, \quad J(u):=-(u-1)^{2}-2 u(u-1)+2 u^{2} \ln u,
$$

$J^{\prime}(u)=-4(u-1)+4 u \ln u$, and

$$
J^{\prime \prime}(u)=4 \ln u<0 \quad(0<u<1) ; \quad J^{\prime \prime}(u)=4 \ln u>0 \quad(u>1) .
$$

It follows that $J_{\min }^{\prime}=J^{\prime}(1)=0, J^{\prime}(u)>0(u \neq 1)$, and $J(u)$ is strictly increasing. Since $J(1)=$ 0 , we have

$$
J(u)<0 \quad(0<u<1) ; \quad J(u)>0 \quad(u>1),
$$

and $g^{\prime \prime}(u)>0(u \neq 1)$. Since $g^{\prime \prime}(1)=\frac{2}{3}>0$, we find $g^{\prime \prime}(u)>0(u>0)$. 
For $0<\lambda \leq 1,0<\lambda_{2}<1$, setting $G(u):=g\left(u^{\lambda}\right) u^{\lambda_{2}-1}(u>0)$, we have $G(u)>0$,

$$
\begin{aligned}
G^{\prime}(u)= & \lambda g^{\prime}\left(u^{\lambda}\right) u^{\lambda+\lambda_{2}-2}+\left(\lambda_{2}-1\right) g\left(u^{\lambda}\right) u^{\lambda_{2}-2}<0, \quad \text { and } \\
G^{\prime \prime}(u)= & \lambda^{2} g^{\prime \prime}\left(u^{\lambda}\right) u^{2 \lambda+\lambda_{2}-3}+\lambda\left(\lambda+\lambda_{2}-2\right) g^{\prime}\left(u^{\lambda}\right) u^{\lambda+\lambda_{2}-3} \\
& +\lambda\left(\lambda_{2}-1\right) g^{\prime}\left(u^{\lambda}\right) u^{\lambda+\lambda_{2}-3}+\left(\lambda_{2}-1\right)\left(\lambda_{2}-2\right) g\left(u^{\lambda}\right) u^{\lambda_{2}-3}>0 .
\end{aligned}
$$

We set $F(x, y):=\frac{\ln (x / y)}{x^{\lambda}-y^{\lambda}}\left(\frac{y}{x}\right)^{\lambda_{2}-1}(x, y>0)$. Since $F(x, y)=\frac{1}{x^{\lambda}} G\left(\frac{y}{x}\right)$, we have

$$
F(x, y)>0, \quad \frac{\partial}{\partial y} F(x, y)<0, \quad \frac{\partial^{2}}{\partial y^{2}} F(x, y)>0 .
$$

Hence, for $x, y>1$, we have

$$
\frac{1}{y} F(\ln x, \ln y)>0, \quad \frac{\partial}{\partial y}\left(\frac{1}{y} F(\ln x, \ln y)\right)<0, \quad \frac{\partial^{2}}{\partial y^{2}}\left(\frac{1}{y} F(\ln x, \ln y)\right)>0 .
$$

Lemma 1 If $f(u)>0, f^{\prime}(u)<0, f^{\prime \prime}(u)>0\left(u>\frac{3}{2}\right)$ and $\int_{\frac{3}{2}}^{\infty} f(u) d u<\infty$, then we have the following Hermite-Hadamard inequality (see [16]):

$$
\int_{k}^{k+1} f(u) d u<f(k)<\int_{k-\frac{1}{2}}^{k+\frac{1}{2}} f(u) d u \quad(k \in \mathbf{N} \backslash\{1\}),
$$

and then

$$
\int_{2}^{\infty} f(u) d u<\sum_{k=2}^{\infty} f(k)<\int_{\frac{3}{2}}^{\infty} f(u) d u
$$

For $|x|,|y| \geq \frac{3}{2}$, define

$$
\begin{gathered}
A_{\xi, \alpha}(x):=|x-\xi|+(x-\xi) \cos \alpha, \\
A_{\eta, \beta}(y)=|y-\eta|+(y-\eta) \cos \beta, \text { and } \\
k(x, y):=\frac{\ln \left(\ln A_{\xi, \alpha}(x) / \ln A_{\eta, \beta}(y)\right)}{\ln ^{\lambda} A_{\xi, \alpha}(x)-\ln ^{\lambda} A_{\eta, \beta}(y)} .
\end{gathered}
$$

We define two weight coefficients as follows:

$$
\begin{aligned}
& \omega\left(\lambda_{2}, m\right):=\sum_{|n|=2}^{\infty} \frac{k(m, n)}{A_{\eta, \beta}(n)} \cdot \frac{\ln ^{\lambda_{1}} A_{\xi, \alpha}(m)}{\ln ^{1-\lambda_{2}} A_{\eta, \beta}(n)}, \quad|m| \in \mathbf{N} \backslash\{1\}, \\
& \varpi\left(\lambda_{1}, n\right):=\sum_{|m|=2}^{\infty} \frac{k(m, n)}{A_{\xi, \alpha}(m)} \cdot \frac{\ln ^{\lambda_{2}} A_{\eta, \beta}(n)}{\ln ^{1-\lambda_{1}} A_{\xi, \alpha}(m)}, \quad|n| \in \mathbf{N} \backslash\{1\},
\end{aligned}
$$

where $\sum_{|j|=2}^{\infty} \cdots=\sum_{j=-2}^{-\infty} \cdots+\sum_{j=2}^{\infty} \cdots(j=m, n)$. 
Lemma 2 We have the inequalities

$$
k_{\beta}\left(\lambda_{1}\right)\left(1-\theta\left(\lambda_{2}, m\right)\right)<\omega\left(\lambda_{2}, m\right)<k_{\beta}\left(\lambda_{1}\right), \quad|m| \in \mathbf{N} \backslash\{1\},
$$

where

$$
\begin{aligned}
\theta\left(\lambda_{2}, m\right) & :=\left[\frac{\lambda}{\pi} \sin \left(\frac{\pi \lambda_{1}}{\lambda}\right)\right]_{0}^{2} \int_{0}^{\frac{\ln [(2+\eta)(1+\cos \beta)]}{\ln A_{\xi, \alpha}(m)}} \frac{\ln u}{u^{\lambda}-1} u^{\lambda_{2}-1} d u \\
& =O\left(\frac{1}{\ln ^{\lambda_{2} / 2} A_{\xi, \alpha}(m)}\right) \in(0,1) .
\end{aligned}
$$

Proof For $|m| \in \mathbf{N} \backslash\{1\}$, let

$$
\begin{aligned}
& k^{(1)}(m, y):=\frac{\ln \ln A_{\xi}(m)-\ln \ln [(y-\eta)(\cos \beta-1)]}{\ln ^{\lambda} A_{\xi}(m)-\ln ^{\lambda}[(y-\eta)(\cos \beta-1)]}, \quad y<-\frac{3}{2}, \\
& k^{(2)}(m, y):=\frac{\ln \ln A_{\xi}(m)-\ln \ln [(y-\eta)(\cos \beta+1)]}{\ln ^{\lambda} A_{\xi}(m)-\ln ^{\lambda}[(y-\eta)(\cos \beta+1)]}, \quad y>\frac{3}{2} .
\end{aligned}
$$

Then the equality

$$
k^{(1)}(m,-y)=\frac{\ln \ln A_{\xi}(m)-\ln \ln [(y+\eta)(1-\cos \beta)]}{\ln ^{\lambda} A_{\xi}(m)-\ln ^{\lambda}[(y+\eta)(1-\cos \beta)]}, \quad y>\frac{3}{2},
$$

yields

$$
\begin{aligned}
\omega\left(\lambda_{2}, m\right)= & \sum_{n=-2}^{-\infty} \frac{k^{(1)}(m, n) \ln ^{\lambda_{1}} A_{\xi, \alpha}(m)}{(n-\eta)(\cos \beta-1) \ln ^{1-\lambda_{2}}[(n-\eta)(\cos \beta-1)]} \\
& +\sum_{n=2}^{\infty} \frac{k^{(2)}(m, n) \ln ^{\lambda_{1}} A_{\xi, \alpha}(m)}{(n-\eta)(1+\cos \beta) \ln ^{1-\lambda_{2}}[(n-\eta)(1+\cos \beta)]} \\
= & \frac{\ln ^{\lambda_{1}} A_{\xi, \alpha}(m)}{1-\cos \beta} \sum_{n=2}^{\infty} \frac{k^{(1)}(m,-n)}{(n+\eta) \ln ^{1-\lambda_{2}}[(n+\eta)(1-\cos \beta)]} \\
& +\frac{\ln ^{\lambda_{1}} A_{\xi, \alpha}(m)}{1+\cos \beta} \sum_{n=2}^{\infty} \frac{k^{(2)}(m, n)}{(n-\eta) \ln ^{1-\lambda_{2}}[(n-\eta)(1+\cos \beta)]}
\end{aligned}
$$

Since $0<\lambda \leq 1,0<\lambda_{2}<1$, by Example 1 we find that, for $y>\frac{3}{2}$,

$$
\begin{aligned}
& \frac{k^{(i)}\left(m,(-1)^{i} y\right)}{\left(y-(-1)^{i} \eta\right) \ln ^{1-\lambda_{2}}\left[\left(y-(-1)^{i} \eta\right)\left(1+(-1)^{i} \cos \beta\right)\right]}>0, \\
& \frac{d}{d y} \frac{k^{(i)}\left(m,(-1)^{i} y\right)}{\left(y-(-1)^{i} \eta\right) \ln ^{1-\lambda_{2}}\left[\left(y-(-1)^{i} \eta\right)\left(1+(-1)^{i} \cos \beta\right)\right]}<0, \\
& \frac{d^{2}}{d y^{2}} \frac{k^{(i)}\left(m,(-1)^{i} y\right)}{\left(y-(-1)^{i} \eta\right) \ln ^{1-\lambda_{2}}\left[\left(y-(-1)^{i} \eta\right)\left(1+(-1)^{i} \cos \beta\right)\right]}>0 \quad(i=1,2),
\end{aligned}
$$

from which it follows that

$$
\frac{k^{(i)}\left(m,(-1)^{i} y\right)}{\left(y-(-1)^{i} \eta\right) \ln ^{1-\lambda_{2}}\left[\left(y-(-1)^{i} \eta\right)\left(1+(-1)^{i} \cos \beta\right)\right]} \quad(i=1,2)
$$


are strictly decreasing and convex in $\left(\frac{3}{2}, \infty\right)$. Then (5) and (11) yield that

$$
\begin{aligned}
\omega\left(\lambda_{2}, m\right)< & \frac{\ln ^{\lambda_{1}} A_{\xi, \alpha}(m)}{1-\cos \beta} \int_{\frac{3}{2}}^{\infty} \frac{k^{(1)}(m,-y)}{(y+\eta) \ln ^{1-\lambda_{2}}[(y+\eta)(1-\cos \beta)]} d y \\
& +\frac{\ln ^{\lambda_{1}} A_{\xi, \alpha}(m)}{1+\cos \beta} \int_{\frac{3}{2}}^{\infty} \frac{k^{(2)}(m, y)}{(y-\eta) \ln ^{1-\lambda_{2}}[(y-\eta)(1+\cos \beta)]} d y
\end{aligned}
$$

Setting $u=\frac{\ln [(y+\eta)(1-\cos \beta)]}{\ln A_{\xi, \alpha}(m)}\left(u=\frac{\ln [(y-\eta)(1+\cos \beta)]}{\ln A_{\xi, \alpha}(m)}\right)$ in the first (second) integral, from Remark 1 we obtain

$$
\begin{aligned}
\omega\left(\lambda_{2}, m\right) & <\left(\frac{1}{1-\cos \beta}+\frac{1}{1+\cos \beta}\right) \int_{0}^{\infty} \frac{\ln u}{u^{\lambda}-1} u^{\lambda_{2}-1} d u \\
& =\frac{2 \csc ^{2} \beta}{\lambda^{2}} \int_{0}^{\infty} \frac{\ln v}{v-1} v^{\left(\lambda_{2} / \lambda\right)-1} d v=\frac{2 \pi^{2} \csc ^{2} \beta}{\lambda^{2} \sin ^{2}\left(\frac{\pi \lambda_{1}}{\lambda}\right)}=k_{\beta}\left(\lambda_{1}\right),
\end{aligned}
$$

by simplifications. Similarly, (5) and (11) also yield that

$$
\begin{aligned}
\omega\left(\lambda_{2}, m\right)> & \frac{\ln ^{\lambda_{1}} A_{\xi, \alpha}(m)}{1-\cos \beta} \int_{2}^{\infty} \frac{k^{(1)}(m,-y)}{(y+\eta) \ln ^{1-\lambda_{2}}[(y+\eta)(1-\cos \beta)]} d y \\
& +\frac{\ln ^{\lambda_{1}} A_{\xi, \alpha}(m)}{1+\cos \beta} \int_{2}^{\infty} \frac{k^{(2)}(m, y)}{(y-\eta) \ln ^{1-\lambda_{2}}[(y-\eta)(1+\cos \beta)]} d y \\
\geq & \left(\frac{1}{1-\cos \beta}+\frac{1}{1+\cos \beta}\right) \int_{\frac{\ln [(2+\eta)(1+\cos \beta)]}{\ln \beta_{\xi, \alpha}(m)}}^{\infty} \frac{\ln u}{u^{\lambda}-1} u^{\lambda_{2}-1} d u \\
= & k_{\beta}\left(\lambda_{1}\right)-2 \csc ^{2} \beta \int_{0}^{\frac{\ln [(2+\eta)(1+\cos \beta)]}{\ln A_{\xi, \alpha}(m)}} \frac{\ln u}{u^{\lambda}-1} u^{\lambda_{2}-1} d u \\
= & k_{\beta}\left(\lambda_{1}\right)\left(1-\theta\left(\lambda_{2}, m\right)\right)>0,
\end{aligned}
$$

where $\theta\left(\lambda_{2}, m\right)(<1)$ is defined in (10). Since

$$
\frac{\ln u}{u^{\lambda}-1} u^{\lambda_{2} / 2} \rightarrow 0 \quad\left(u \rightarrow 0^{+}\right) ; \quad \frac{\ln u}{u^{\lambda}-1} u^{\lambda_{2} / 2} \rightarrow \frac{1}{\lambda} \quad(u \rightarrow 1),
$$

there exists a positive constant $C$ such that $\frac{\ln u}{u^{\lambda}-1} u^{\lambda_{2} / 2} \leq C(0<u \leq 1)$. Then for $A_{\xi, \alpha}(m) \geq$ $(2+\eta)(1+\cos \beta)$, we have

$$
\begin{aligned}
0 & <\theta\left(\lambda_{2}, m\right) \leq C\left[\frac{\lambda}{\pi} \sin \left(\frac{\pi \lambda_{1}}{\lambda}\right)\right]^{2} \int_{0}^{\frac{\ln [(2+\eta)(1+\cos \beta)]}{\ln A_{\xi, \alpha}(m)}} u^{\frac{\lambda_{2}}{2}-1} d u \\
& =\frac{2 C}{\lambda_{2}}\left[\frac{\lambda}{\pi} \sin \left(\frac{\pi \lambda_{1}}{\lambda}\right)\right]^{2}\left\{\frac{\ln [(2+\eta)(1+\cos \beta)]}{\ln A_{\xi, \alpha}(m)}\right\}^{\frac{\lambda}{2}} .
\end{aligned}
$$

Hence, (9) and (10) are valid.

Similarly, we have the following:

Lemma 3 For $0<\lambda \leq 1,0<\lambda_{1}<1$, we have the inequalities

$$
k_{\alpha}\left(\lambda_{1}\right)\left(1-\tilde{\theta}\left(\lambda_{1}, n\right)\right)<\varpi\left(\lambda_{1}, n\right)<k_{\alpha}\left(\lambda_{1}\right), \quad|n| \in \mathbf{N} \backslash\{1\},
$$


where

$$
\begin{aligned}
\tilde{\theta}\left(\lambda_{1}, n\right): & :=\left[\frac{\lambda}{\pi} \sin \left(\frac{\pi \lambda_{1}}{\lambda}\right)\right]^{2} \int_{0}^{\frac{\ln ([2+\xi)(1+\cos \alpha)]}{\ln A_{\eta, \beta}(n)}} \frac{\ln u}{u^{\lambda}-1} u^{\lambda_{1}-1} d u \\
& =O\left(\frac{1}{\ln ^{\lambda_{1} / 2} A_{\eta, \beta}(n)}\right) \in(0,1) .
\end{aligned}
$$

Lemma 4 If $(\zeta, \gamma)=(\xi, \alpha)($ or $(\eta, \beta)), \rho>0$, then we have

$$
H_{\rho}(\zeta, \gamma):=\sum_{|k|=2}^{\infty} \frac{\ln ^{-1-\rho} A_{\zeta, \gamma}(k)}{A_{\zeta, \gamma}(k)}=\frac{1}{\rho}\left(2 \csc ^{2} \gamma+o(1)\right) \quad\left(\rho \rightarrow 0^{+}\right) .
$$

Proof By (5) we obtain

$$
\begin{aligned}
H_{\rho}(\varsigma, \gamma) & =\sum_{k=-2}^{-\infty} \frac{\ln ^{-1-\rho}[(k-\varsigma)(\cos \gamma-1)]}{(k-\varsigma)(\cos \gamma-1)}+\sum_{k=2}^{\infty} \frac{\ln ^{-1-\rho}[(k-\varsigma)(\cos \gamma+1)]}{(k-\varsigma)(\cos \gamma+1)} \\
& =\sum_{k=2}^{\infty}\left\{\frac{\ln ^{-1-\rho}[(k+\varsigma)(1-\cos \gamma)]}{(k-\varsigma)(1-\cos \gamma)}+\frac{\ln ^{-1-\rho}[(k-\varsigma)(\cos \gamma+1)]}{(k-\varsigma)(\cos \gamma+1)}\right\} \\
& <\int_{\frac{3}{2}}^{\infty}\left\{\frac{\ln ^{-1-\rho}[(y+\varsigma)(1-\cos \gamma)]}{(y-\varsigma)(1-\cos \gamma)}+\frac{\ln ^{-1-\rho}[(y-\varsigma)(\cos \gamma+1)]}{(y-\varsigma)(\cos \gamma+1)}\right\} d y \\
& =\frac{1}{\rho}\left\{\frac{\ln ^{-\rho}\left[\left(\frac{3}{2}+\varsigma\right)(1-\cos \gamma)\right]}{1-\cos \gamma}+\frac{\ln ^{-\rho}\left[\left(\frac{3}{2}-\varsigma\right)(1+\cos \gamma)\right]}{1+\cos \gamma}\right\} \\
& =\frac{1}{\rho}\left(\frac{1}{1-\cos \gamma}+\frac{1}{1+\cos \gamma}+o_{1}(1)\right)=\frac{1}{\rho}\left(2 \csc ^{2} \gamma+o_{1}(1)\right) \quad\left(\rho \rightarrow 0^{+}\right)
\end{aligned}
$$

and

$$
\begin{aligned}
H_{\rho}(\varsigma, \gamma) & =\sum_{k=2}^{\infty}\left\{\frac{\ln ^{-1-\rho}[(k+\varsigma)(1-\cos \gamma)]}{(k-\varsigma)(1-\cos \gamma)}+\frac{\ln ^{-1-\rho}[(k-\varsigma)(\cos \gamma+1)]}{(k-\varsigma)(\cos \gamma+1)}\right\} \\
& >\int_{2}^{\infty}\left\{\frac{\ln ^{-1-\rho}[(y+\varsigma)(1-\cos \gamma)]}{(y-\varsigma)(1-\cos \gamma)}+\frac{\ln ^{-1-\rho}[(y-\varsigma)(\cos \gamma+1)]}{(y-\varsigma)(\cos \gamma+1)}\right\} d y \\
& =\frac{1}{\rho}\left\{\frac{\ln ^{-\rho}[(2+\varsigma)(1-\cos \gamma)]}{1-\cos \gamma}+\frac{\ln ^{-\rho}[(2-\varsigma)(1+\cos \gamma)]}{1+\cos \gamma}\right\} \\
& =\frac{1}{\rho}\left(\frac{1}{1-\cos \gamma}+\frac{1}{1+\cos \gamma}+o_{2}(1)\right)=\frac{1}{\rho}\left(2 \csc ^{2} \gamma+o_{2}(1)\right) \quad\left(\rho \rightarrow 0^{+}\right) .
\end{aligned}
$$

Therefore, (15) is valid.

\section{Main results and a few particular cases}

Theorem 1 Suppose that $0<p<1, \frac{1}{p}+\frac{1}{q}=1$,

$$
k\left(\lambda_{1}\right):=k_{\beta}^{1 / p}\left(\lambda_{1}\right) k_{\alpha}^{1 / q}\left(\lambda_{1}\right)=\frac{2 \pi^{2} \csc ^{2 / p} \beta \csc ^{2 / q} \alpha}{\left[\lambda \sin \left(\frac{\pi \lambda_{1}}{\lambda}\right)\right]^{2}} .
$$




$$
\begin{aligned}
& \text { If } a_{m}, b_{n} \geq 0(|m|,|n| \in \mathbf{N} \backslash\{1\}), \text { satisfy } \\
& \quad 0<\sum_{|m|=2}^{\infty} \frac{\ln ^{p\left(1-\lambda_{1}\right)-1} A_{\xi, \alpha}(m)}{\left(A_{\xi, \alpha}(m)\right)^{1-p}} a_{m}^{p}<\infty, \quad 0<\sum_{|n|=2}^{\infty} \frac{\ln ^{q\left(1-\lambda_{2}\right)-1} A_{\eta, \beta}(n)}{\left(A_{\eta, \beta}(n)\right)^{1-q}} b_{n}^{q}<\infty,
\end{aligned}
$$

then for

$$
\begin{aligned}
\theta\left(\lambda_{2}, m\right) & =\left[\frac{\lambda}{\pi} \sin \left(\frac{\pi \lambda_{1}}{\lambda}\right)\right]^{2} \int_{0}^{\frac{\ln [(2+\eta)(1+\cos \beta)]}{\ln A_{\xi, \alpha}(m)}} \frac{\ln u}{u^{\lambda}-1} u^{\lambda_{2}-1} d u \\
& =O\left(\frac{1}{\ln ^{\lambda_{2} / 2} A_{\xi, \alpha}(m)}\right) \in(0,1),
\end{aligned}
$$

we obtain the following equivalent reverse Mulholland-type inequalities:

$$
\begin{aligned}
& I:=\sum_{|n|=2}^{\infty} \sum_{|m|=2}^{\infty} \frac{\ln \left(\ln A_{\xi, \alpha}(m) / \ln A_{\eta, \beta}(n)\right)}{\ln ^{\lambda} A_{\xi, \alpha}(m)-\ln ^{\lambda} A_{\eta, \beta}(n)} a_{m} b_{n} \\
& >\frac{2 \pi^{2} \csc ^{2 / p} \beta \csc ^{2 / q} \alpha}{\left[\lambda \sin \left(\frac{\pi \lambda_{1}}{\lambda}\right)\right]^{2}} \\
& \times\left[\sum_{|m|=2}^{\infty}\left(1-\theta\left(\lambda_{2}, m\right)\right) \frac{\ln ^{p\left(1-\lambda_{1}\right)-1} A_{\xi, \alpha}(m)}{\left(A_{\xi, \alpha}(m)\right)^{1-p}} a_{m}^{p}\right]^{\frac{1}{p}} \\
& \times\left[\sum_{|n|=2}^{\infty} \frac{\ln ^{q\left(1-\lambda_{2}\right)-1} A_{\eta, \beta}(n)}{\left(A_{\eta, \beta}(n)\right)^{1-q}} b_{n}^{q}\right]^{\frac{1}{q}} \\
& J_{1}:=\left\{\sum_{|n|=2}^{\infty} \frac{\ln ^{p \lambda_{2}-1} A_{\eta, \beta}(n)}{A_{\eta, \beta}(n)}\left[\sum_{|m|=2}^{\infty} \frac{\ln \left(\ln A_{\xi, \alpha}(m) / \ln A_{\eta, \beta}(n)\right)}{\ln ^{\lambda} A_{\xi, \alpha}(m)-\ln ^{\lambda} A_{\eta, \beta}(n)} a_{m}\right]^{p}\right\}^{\frac{1}{p}} \\
& >\frac{2 \pi^{2} \csc ^{2 / p} \beta \csc ^{2 / q} \alpha}{\left[\lambda \sin \left(\frac{\pi \lambda_{1}}{\lambda}\right)\right]^{2}}\left[\sum_{|m|=2}^{\infty}\left(1-\theta\left(\lambda_{2}, m\right)\right) \frac{\ln ^{p\left(1-\lambda_{1}\right)-1} A_{\xi, \alpha}(m)}{\left(A_{\xi, \alpha}(m)\right)^{1-p}} a_{m}^{p}\right]^{\frac{1}{p}} \text {, } \\
& J_{2}:=\left[\sum_{|m|=2}^{\infty} \frac{\ln ^{q \lambda_{1}-1} A_{\xi, \alpha}(m)}{\left(1-\theta\left(\lambda_{2}, m\right)\right)^{q-1} A_{\xi, \alpha}(m)}\left(\sum_{|n|=2}^{\infty} \frac{\ln \left(\ln A_{\xi, \alpha}(m) / \ln A_{\eta, \beta}(n)\right)}{\ln ^{\lambda} A_{\xi, \alpha}(m)-\ln ^{\lambda} A_{\eta, \beta}(n)} b_{n}\right)^{q}\right]^{\frac{1}{q}} \\
& >\frac{2 \pi^{2} \csc ^{2 / p} \beta \csc ^{2 / q} \alpha}{\left[\lambda \sin \left(\frac{\pi \lambda_{1}}{\lambda}\right)\right]^{2}}\left[\sum_{|n|=2}^{\infty} \frac{\ln ^{q\left(1-\lambda_{2}\right)-1} A_{\eta, \beta}(n)}{\left(A_{\eta, \beta}(n)\right)^{1-q}} b_{n}^{q}\right]^{\frac{1}{q}} \text {. }
\end{aligned}
$$

Particularly, (i) for $\alpha=\beta=\frac{\pi}{2}, \xi, \eta \in\left[0, \frac{1}{2}\right]$, setting

$$
\begin{aligned}
\theta_{1}\left(\lambda_{2}, m\right) & :=\left[\frac{\lambda}{\pi} \sin \left(\frac{\pi \lambda_{1}}{\lambda}\right)\right]^{2} \int_{0}^{\frac{\ln (2+\eta)}{\ln |m-\xi|}} \frac{\ln u}{u^{\lambda}-1} u^{\lambda_{2}-1} d u \\
& =O\left(\frac{1}{\ln ^{\lambda_{2} / 2}|m-\xi|}\right) \in(0,1),
\end{aligned}
$$


we have the following equivalent reverse Mulholland-type inequalities:

$$
\begin{aligned}
& \sum_{|n|=2}^{\infty} \sum_{|m|=2}^{\infty} \frac{\ln (|m-\xi| /|n-\eta|)}{\ln ^{\lambda}|m-\xi|-\ln ^{\lambda}|n-\eta|} a_{m} b_{n} \\
& >\frac{2 \pi^{2}}{\left[\lambda \sin \left(\frac{\pi \lambda_{1}}{\lambda}\right)\right]^{2}} \\
& \times\left[\sum_{|m|=2}^{\infty}\left(1-\theta_{1}\left(\lambda_{2}, m\right)\right) \frac{\ln ^{p\left(1-\lambda_{1}\right)-1}|m-\xi|}{|m-\xi|^{1-p}} a_{m}^{p}\right]^{\frac{1}{p}} \\
& \times\left[\sum_{|n|=2}^{\infty} \frac{\ln ^{q\left(1-\lambda_{2}\right)-1}|n-\eta|}{|n-\eta|^{1-q}} b_{n}^{q}\right]^{\frac{1}{q}} \\
& {\left[\sum_{|n|=2}^{\infty} \frac{\ln ^{p \lambda_{2}-1}|n-\eta|}{|n-\eta|}\left(\sum_{|m|=2}^{\infty} \frac{\ln (|m-\xi| /|n-\eta|)}{\ln ^{\lambda}|m-\xi|-\ln ^{\lambda}|n-\eta|} a_{m}\right)^{p}\right]^{\frac{1}{p}}} \\
& >\frac{2 \pi^{2}}{\left[\lambda \sin \left(\frac{\pi \lambda_{1}}{\lambda}\right)\right]^{2}}\left[\sum_{|m|=2}^{\infty}\left(1-\theta_{1}\left(\lambda_{2}, m\right)\right) \frac{\ln p\left(1-\lambda_{1}\right)-1}{|m-\xi|} a_{m}^{p}\right]^{\frac{1}{p}}, \\
& {\left[\sum_{|m|=2}^{\infty} \frac{\ln ^{q \lambda_{1}-1}|m-\xi|}{\left(1-\theta_{1}\left(\lambda_{2}, m\right)\right)^{q-1}|m-\xi|}\left(\sum_{|n|=2}^{\infty} \frac{\ln (|m-\xi| /|n-\eta|) a_{m}}{\ln ^{\lambda}|m-\xi|-\ln ^{\lambda}|n-\eta|} b_{n}\right)^{q}\right]^{\frac{1}{q}}} \\
& >\frac{2 \pi^{2}}{\left[\lambda \sin \left(\frac{\pi \lambda_{1}}{\lambda}\right)\right]^{2}}\left[\sum_{|n|=2}^{\infty} \frac{\ln ^{q\left(1-\lambda_{2}\right)-1}|n-\eta|}{|n-\eta|^{1-q}} b_{n}^{q}\right]^{\frac{1}{q}} .
\end{aligned}
$$

(ii) For $\xi=\eta=0, \alpha, \beta \in\left[\arccos \frac{1}{3}, \frac{\pi}{2}\right]$, setting

$$
\begin{aligned}
\theta_{2}\left(\lambda_{2}, m\right) & :=\left[\frac{\lambda}{\pi} \sin \left(\frac{\pi \lambda_{1}}{\lambda}\right)\right]^{2} \int_{0}^{\frac{\ln 2(1+\cos \beta)}{\ln (|m|+m \cos \alpha)}} \frac{\ln u}{u^{\lambda}-1} u^{\lambda_{2}-1} d u \\
& =O\left(\frac{1}{\ln ^{\lambda_{2} / 2} A_{\xi, \alpha}(m)}\right) \in(0,1),
\end{aligned}
$$

we have the following equivalent reverse Mulholland-type inequalities:

$$
\begin{aligned}
\sum_{|n|=2}^{\infty} \sum_{|m|=2}^{\infty} \frac{\ln [\ln (|m|+m \cos \alpha) / \ln (|n|+n \cos \beta)]}{\ln ^{\lambda}(|m|+m \cos \alpha)-\ln ^{\lambda}(|n|+n \cos \beta)} a_{m} b_{n} \\
>\frac{2 \pi^{2} \csc ^{2 / p} \beta \csc ^{2 / q} \alpha}{\left[\lambda \sin \left(\frac{\pi \lambda_{1}}{\lambda}\right)\right]^{2}} \\
\times\left[\sum_{|m|=2}^{\infty}\left(1-\theta_{2}\left(\lambda_{2}, m\right)\right) \frac{\ln ^{p\left(1-\lambda_{1}\right)-1}(|m|+m \cos \alpha)}{(|m|+m \cos \alpha)^{1-p}} a_{m}^{p}\right]^{\frac{1}{p}} \\
\times\left[\sum_{|n|=2}^{\infty} \frac{\ln ^{q\left(1-\lambda_{2}\right)-1}(|n|+n \cos \beta)}{(|n|+n \cos \beta)^{1-q}} b_{n}^{q}\right]^{\frac{1}{q}},
\end{aligned}
$$




$$
\begin{aligned}
& \left\{\sum_{|n|=2}^{\infty} \frac{\ln ^{p \lambda_{2}-1}(|n|+n \cos \beta)}{|n|+n \cos \beta}\left[\sum_{|m|=2}^{\infty} \frac{\ln [\ln (|m|+m \cos \alpha) / \ln (|n|+n \cos \beta)]}{\ln ^{\lambda}(|m|+m \cos \alpha)-\ln ^{\lambda}(|n|+n \cos \beta)} a_{m}\right]^{p}\right\}^{\frac{1}{p}} \\
& >\frac{2 \pi^{2} \csc ^{2 / p} \beta \csc ^{2 / q} \alpha}{\left[\lambda \sin \left(\frac{\pi \lambda_{1}}{\lambda}\right)\right]^{2}}\left[\sum_{|m|=2}^{\infty}\left(1-\theta_{2}\left(\lambda_{2}, m\right)\right) \frac{\ln ^{p\left(1-\lambda_{1}\right)-1}(|m|+m \cos \alpha)}{(|m|+m \cos \alpha)^{1-p}} a_{m}^{p}\right]^{\frac{1}{p}} \\
& {\left[\sum_{|m|=2}^{\infty} \frac{\ln ^{q \lambda_{1}-1}(|m|+m \cos \alpha)}{\left(1-\theta_{2}\left(\lambda_{2}, m\right)\right)^{q-1}(|m|+m \cos \alpha)}\right.} \\
& \left.\quad \times\left(\sum_{|n|=2}^{\infty} \frac{\ln [\ln (|m|+m \cos \alpha) / \ln (|n|+n \cos \beta)]}{\ln ^{\lambda}(|m|+m \cos \alpha)-\ln ^{\lambda}(|n|+n \cos \beta)} b_{n}\right)^{q}\right]^{\frac{1}{q}} \\
& >\frac{2 \pi^{2} \csc ^{2 / p} \beta \csc ^{2 / q} \alpha}{\left[\lambda \sin ^{2}\left(\frac{\pi \lambda_{1}}{\lambda}\right)\right]^{2}}\left[\sum_{|n|=2}^{\infty} \frac{\ln ^{q\left(1-\lambda_{2}\right)-1}(|n|+n \cos \beta)}{(|n|+n \cos \beta)^{1-q}} b_{n}^{q}\right]^{\frac{1}{q}}
\end{aligned}
$$

Proof Applying the reverse Hölder inequality with weight (see [17]) and (8), we find

$$
\begin{aligned}
\left(\sum_{|m|=2}^{\infty} k(m, n) a_{m}\right)^{p} & \\
= & \left\{\sum_{|m|=2}^{\infty} k(m, n)\left[\frac{\left(A_{\xi, \alpha}(m)\right)^{\frac{1}{q}} \ln ^{\frac{1-\lambda_{1}}{q}} A_{\xi, \alpha}(m)}{\ln ^{\frac{1-\lambda_{2}}{p}} A_{\eta, \beta}(n)} a_{m}\right]\left[\frac{\ln ^{\frac{1-\lambda_{2}}{p}} A_{\eta, \beta}(n)}{\left(A_{\xi, \alpha}(m)\right)^{\frac{1}{q}} \ln ^{\frac{1-\lambda_{1}}{q}} A_{\xi, \alpha}(m)}\right]\right\}^{p} \\
\geq & \sum_{|m|=2}^{\infty} k(m, n) \frac{\left(A_{\xi, \alpha}(m)\right)^{\frac{p}{q}} \ln ^{\frac{\left(1-\lambda_{1}\right) p}{q}} A_{\xi, \alpha}(m)}{\ln ^{1-\lambda_{2}} A_{\eta, \beta}(n)} a_{m}^{p} \\
& \times\left[\sum_{|m|=2}^{\infty} k(m, n) \frac{\ln ^{\frac{\left(1-\lambda_{2}\right) q}{p}} A_{\eta, \beta}(n)}{A_{\xi, \alpha}(m) \ln ^{1-\lambda_{1}} A_{\xi, \alpha}(m)}\right]^{p-1} \\
= & \frac{\left(\varpi\left(\lambda_{1}, n\right)\right)^{p-1} A_{\eta, \beta}(n)}{\ln ^{p \lambda_{2}-1} A_{\eta, \beta}(n)} \sum_{|m|=2}^{\infty} k(m, n) \frac{\left(A_{\xi, \alpha}(m)\right)^{\frac{p}{q}} \ln ^{\frac{\left(1-\lambda_{1}\right) p}{q}} A_{\xi, \alpha}(m)}{A_{\eta, \beta}(n) \ln ^{1-\lambda_{2}} A_{\eta, \beta}(n)} a_{m}^{p} .
\end{aligned}
$$

Then since $0<p<1$, by (13) this yields

$$
\begin{aligned}
J & >k_{\alpha}^{1 / q}\left(\lambda_{1}\right)\left[\sum_{|n|=2}^{\infty} \sum_{|m|=2}^{\infty} k(m, n) \frac{\left(A_{\xi, \alpha}(m)\right)^{\frac{p}{q}} \ln ^{\frac{\left(1-\lambda_{1}\right) p}{q}} A_{\xi, \alpha}(m)}{A_{\eta, \beta}(n) \ln ^{1-\lambda_{2}} A_{\eta, \beta}(n)} a_{m}^{p}\right]^{\frac{1}{p}} \\
& =k_{\alpha}^{1 / q}\left(\lambda_{1}\right)\left[\sum_{|m|=2}^{\infty} \sum_{|n|=2}^{\infty} k(m, n) \frac{\left(A_{\xi, \alpha}(m)\right)^{\frac{p}{q}} \ln ^{\frac{\left(1-\lambda_{1}\right) p}{q}} A_{\xi, \alpha}(m)}{A_{\eta, \beta}(n) \ln ^{1-\lambda_{2}} A_{\eta, \beta}(n)} a_{m}^{p}\right]^{\frac{1}{p}} \\
& =k_{\alpha}^{1 / q}\left(\lambda_{1}\right)\left[\sum_{|m|=2}^{\infty} \omega\left(\lambda_{2}, m\right) \frac{n^{p\left(1-\lambda_{1}\right)-1} A_{\xi, \alpha}(m)}{\left(A_{\xi, \alpha}(m)\right)^{1-p}} a_{m}^{p}\right]^{\frac{1}{p}} .
\end{aligned}
$$

Combining (9) and (16), we obtain (18). 
Using the reverse Hölders inequality again, we obtain

$$
\begin{aligned}
I & =\sum_{|n|=2}^{\infty}\left[\frac{\left(A_{\eta, \beta}(n)\right)^{\frac{-1}{p}}}{\ln ^{\frac{1}{p}-\lambda_{2}} A_{\eta, \beta}(n)} \sum_{|m|=2}^{\infty} k(m, n) a_{m}\right]\left[\frac{\ln ^{\frac{1}{p}-\lambda_{2}} A_{\eta, \beta}(n)}{\left(A_{\eta, \beta}(n)\right)^{\frac{-1}{p}}} b_{n}\right] \\
& \geq J_{1}\left[\sum_{|n|=2}^{\infty} \frac{\ln ^{q\left(1-\lambda_{2}\right)-1} A_{\eta, \beta}(n)}{\left(A_{\eta, \beta}(n)\right)^{1-q}} b_{n}^{q}\right]^{\frac{1}{q}} .
\end{aligned}
$$

Then by (18) we obtain (17).

On the other-hand, assuming that (17) is valid, letting

$$
b_{n}:=\frac{\ln ^{p \lambda_{2}-1} A_{\eta, \beta}(n)}{A_{\eta, \beta}(n)}\left(\sum_{|m|=2}^{\infty} k(m, n) a_{m}\right)^{p-1}, \quad|n| \in \mathbf{N} \backslash\{1\},
$$

we find

$$
J_{1}=\left[\sum_{|n|=2}^{\infty} \frac{\ln ^{q\left(1-\lambda_{2}\right)-1} A_{\eta, \beta}(n)}{\left(A_{\eta, \beta}(n)\right)^{1-q}} b_{n}^{q}\right]^{\frac{1}{p}} .
$$

By (26) it follows that $J_{1}>0$. If $J_{1}=\infty$, then (19) is trivially valid; if $J_{1}<\infty$, then by (17) we have

$$
\begin{aligned}
& \sum_{|n|=2}^{\infty} \frac{\ln ^{q\left(1-\lambda_{2}\right)-1} A_{\eta, \beta}(n)}{\left(A_{\eta, \beta}(n)\right)^{1-q}} b_{n}^{q} \\
& \quad=J_{1}^{p}=I \\
& \quad>k\left(\lambda_{1}\right)\left[\sum_{|m|=2}^{\infty}\left(1-\theta\left(\lambda_{2}, m\right)\right) \frac{\ln ^{p\left(1-\lambda_{1}\right)-1} A_{\xi, \alpha}(m)}{\left(A_{\xi, \alpha}(m)\right)^{1-p}} a_{m}^{p}\right]^{\frac{1}{p}}\left[\sum_{|n|=2}^{\infty} \frac{\ln ^{q\left(1-\lambda_{2}\right)-1} A_{\eta, \beta}(n)}{\left(A_{\eta, \beta}(n)\right)^{1-q}} b_{n}^{q}\right]^{\frac{1}{q}}, \\
& J_{1}=\left[\sum_{|n|=2}^{\infty} \frac{\ln ^{q\left(1-\lambda_{2}\right)-1} A_{\eta, \beta}(n)}{\left(A_{\eta, \beta}(n)\right)^{1-q}} b_{n}^{q}\right]^{\frac{1}{p}}>k\left(\lambda_{1}\right)\left[\sum_{|m|=2}^{\infty}\left(1-\theta\left(\lambda_{2}, m\right)\right) \frac{\ln ^{p\left(1-\lambda_{1}\right)-1} A_{\xi, \alpha}(m)}{\left(A_{\xi, \alpha}(m)\right)^{1-p}} a_{m}^{p}\right]^{\frac{1}{p}} .
\end{aligned}
$$

Thus (18) is valid, which is equivalent to (17).

We further prove that (19) is equivalent to (17). Using the reverse Hölders inequality, we have

$$
\begin{aligned}
I= & \sum_{|m|=2}^{\infty}\left[\left(1-\theta\left(\lambda_{2}, m\right)\right)^{\frac{1}{p}} \frac{\ln ^{\frac{1}{q}-\lambda_{1}} A_{\xi, \alpha}(m)}{\left(A_{\xi, \alpha}(m)\right)^{\frac{-1}{q}}} a_{m}\right] \\
& \times\left[\frac{n^{\frac{-1}{q}+\lambda_{1}} A_{\xi, \alpha}(m)}{\left(1-\theta\left(\lambda_{2}, m\right)\right)^{\frac{1}{p}}\left(A_{\xi, \alpha}(m)\right)^{\frac{1}{q}}} \sum_{|n|=2}^{\infty} k(m, n) b_{n}\right] \\
\geq & {\left[\sum_{|m|=2}^{\infty}\left(1-\theta\left(\lambda_{2}, m\right)\right) \frac{\ln ^{p\left(1-\lambda_{1}\right)-1} A_{\xi, \alpha}(m)}{\left(A_{\xi, \alpha}(m)\right)^{1-p}} a_{m}^{p}\right]^{\frac{1}{p}} J_{2}, }
\end{aligned}
$$

and then (19) is valid by (17). 
On the other-hand, assuming that (17) is valid, we set

$$
a_{m}:=\frac{\ln ^{q \lambda_{1}-1} A_{\xi, \alpha}(m)}{\left(1-\theta\left(\lambda_{2}, m\right)\right)^{q-1} A_{\xi, \alpha}(m)}\left(\sum_{|n|=2}^{\infty} \frac{\ln \left(\ln A_{\xi, \alpha}(m) / \ln A_{\eta, \beta}(n)\right)}{\ln ^{\lambda} A_{\xi, \alpha}(m)-\ln ^{\lambda} A_{\eta, \beta}(n)} b_{n}\right)^{q-1}, \quad m \in \mathbf{N} \backslash\{1\},
$$

and find

$$
J_{2}=\left[\sum_{|m|=2}^{\infty}\left(1-\theta\left(\lambda_{2}, m\right)\right) \frac{\ln ^{p\left(1-\lambda_{1}\right)-1} A_{\xi, \alpha}(m)}{\left(A_{\xi, \alpha}(m)\right)^{1-p}} a_{m}^{p}\right]^{\frac{1}{q}}
$$

If $J_{2}=0$, then (19) is impossible, so that $J_{2}>0$. If $J_{2}=\infty$, then (19) is trivially valid; if $J_{2}<\infty$, then by (17) we have

$$
\begin{aligned}
& \sum_{|m|=2}^{\infty}\left(1-\theta\left(\lambda_{2}, m\right)\right) \frac{\ln ^{p\left(1-\lambda_{1}\right)-1} A_{\xi, \alpha}(m)}{\left(A_{\xi, \alpha}(m)\right)^{1-p}} a_{m}^{p} \\
& \quad=J_{2}^{q}=I \\
& \quad>k\left(\lambda_{1}\right)\left[\sum_{|m|=2}^{\infty}\left(1-\theta\left(\lambda_{2}, m\right)\right) \frac{\ln ^{p\left(1-\lambda_{1}\right)-1} A_{\xi, \alpha}(m)}{\left(A_{\xi, \alpha}(m)\right)^{1-p}} a_{m}^{p}\right]^{\frac{1}{p}}\left[\sum_{|n|=2}^{\infty} \frac{\ln ^{q\left(1-\lambda_{2}\right)-1} A_{\eta, \beta}(n)}{\left(A_{\eta, \beta}(n)\right)^{1-q}} b_{n}^{q}\right]^{\frac{1}{q}}, \\
& {\left[\sum_{|m|=2}^{\infty}\left(1-\theta\left(\lambda_{2}, m\right)\right) \frac{\ln ^{p\left(1-\lambda_{1}\right)-1} A_{\xi, \alpha}(m)}{\left(A_{\xi, \alpha}(m)\right)^{1-p}} a_{m}^{p}\right]^{\frac{1}{q}}} \\
& \quad=J_{2} \\
& \quad>k\left(\lambda_{1}\right)\left[\sum_{|n|=2}^{\infty} \frac{\ln ^{q\left(1-\lambda_{2}\right)-1} A_{\eta, \beta}(n)}{\left(A_{\eta, \beta}(n)\right)^{1-q}} b_{n}^{q}\right]^{\frac{1}{q}} .
\end{aligned}
$$

Thus (19) is valid, which is equivalent to (17).

Hence, inequalities (17), (18), and (19) are equivalent.

Theorem 2 Under the assumptions in Theorem 1,

$$
k\left(\lambda_{1}\right)=\frac{2 \pi^{2} \csc ^{2 / p} \beta \csc ^{2 / q} \alpha}{\left[\lambda \sin \left(\frac{\pi \lambda_{1}}{\lambda}\right)\right]^{2}}
$$

is the best possible constant factor in (17), (18), and (19).

Proof For $0<\varepsilon<\min \left\{p \lambda_{1}, p\left(1-\lambda_{2}\right)\right\}$, we set $\tilde{\lambda}_{1}=\lambda_{1}-\frac{\varepsilon}{p}(\in(0,1)), \tilde{\lambda}_{2}=\lambda_{2}+\frac{\varepsilon}{p}(\in(0,1))$, and

$$
\begin{aligned}
& \tilde{a}_{m}:=\frac{\ln ^{\lambda_{1}-\frac{\varepsilon}{p}-1} A_{\xi, \alpha}(m)}{A_{\xi, \alpha}(m)}=\frac{\ln ^{\tilde{\lambda}_{1}-1} A_{\xi, \alpha}(m)}{A_{\xi, \alpha}(m)} \quad(|m| \in \mathbf{N} \backslash\{1\}), \\
& \tilde{b}_{n}:=\frac{\ln ^{\lambda_{2}-\frac{\varepsilon}{q}-1} A_{\eta, \beta}(n)}{A_{\eta, \beta}(n)}=\frac{\ln ^{\tilde{\lambda}_{2}-\varepsilon-1} A_{\eta, \beta}(n)}{A_{\eta, \beta}(n)} \quad(|n| \in \mathbf{N} \backslash\{1\}) .
\end{aligned}
$$


By (15) and (13) we find

$$
\begin{aligned}
\tilde{I}_{2} & :=\left[\sum_{|m|=2}^{\infty}\left(1-\theta\left(\lambda_{2}, m\right)\right) \frac{\ln ^{p\left(1-\lambda_{1}\right)-1} A_{\xi, \alpha}(m)}{\left(A_{\xi, \alpha}(m)\right)^{1-p}} \tilde{a}_{m}^{p}\right]^{\frac{1}{p}}\left[\sum_{|n|=2}^{\infty} \frac{\ln ^{q\left(1-\lambda_{2}\right)-1} A_{\eta, \beta}(n)}{\left(A_{\eta, \beta}(n)\right)^{1-q}} \tilde{b}_{n}^{q}\right]^{\frac{1}{q}} \\
& =\left[\sum_{|m|=2}^{\infty} \frac{\ln ^{-1-\varepsilon} A_{\xi, \alpha}(m)}{A_{\xi, \alpha}(m)}-\sum_{|m|=2}^{\infty} \frac{O\left(\ln ^{-1-\left(\frac{\lambda_{2}}{2}+\varepsilon\right)} A_{\xi, \alpha}(m)\right)}{A_{\xi, \alpha}(m)}\right]^{\frac{1}{p}}\left[\sum_{|n|=2}^{\infty} \frac{\ln ^{-1-\varepsilon} A_{\eta, \beta}(n)}{A_{\eta, \beta}(n)}\right]^{\frac{1}{q}} \\
& =\frac{1}{\varepsilon}\left(2 \csc ^{2} \alpha+o(1)-\varepsilon O(1)\right)^{\frac{1}{p}}\left(2 \csc ^{2} \beta+\tilde{o}(1)\right)^{\frac{1}{q}}\left(\varepsilon \rightarrow 0^{+}\right), \\
\tilde{I}= & \sum_{|n|=2}^{\infty} \sum_{|m|=2}^{\infty} k(m, n) \tilde{a}_{m} \tilde{b}_{n}=\sum_{|m|=2}^{\infty} \sum_{|n|=2}^{\infty} k(m, n) \frac{\ln ^{\tilde{\lambda}_{1}-1} A_{\xi, \alpha}(m)}{A_{\xi, \alpha}(m)} \frac{\ln ^{\tilde{\lambda}_{2}-\varepsilon-1} A_{\eta, \beta}(n)}{A_{\eta, \beta}(n)} \\
& =\sum_{|n|=2}^{\infty} \varpi\left(\tilde{\lambda}_{1}, n\right) \frac{\ln ^{-1-\varepsilon} A_{\eta, \beta}(n)}{A_{\eta, \beta}(n)}<k_{\alpha}\left(\tilde{\lambda}_{1}\right) \sum_{|n|=2}^{\infty} \frac{\ln ^{-1-\varepsilon} A_{\eta, \beta}(n)}{A_{\eta, \beta}(n)} \\
& =\frac{1}{\varepsilon} k_{\alpha}\left(\tilde{\lambda}_{1}\right)\left(2 \csc ^{2} \beta+o(1)\right) .
\end{aligned}
$$

If there exists a positive number $k \geq k\left(\lambda_{1}\right)$ such that (17) is still valid when replacing $k\left(\lambda_{1}\right)$ by $k$, then, in particular, we have

$$
\varepsilon \tilde{I}=\varepsilon \sum_{|m|=2}^{\infty} \sum_{|n|=2}^{\infty} k(m, n) \tilde{a}_{m} \tilde{b}_{n}>\varepsilon k \tilde{I}_{2} .
$$

We obtain from the previous results that

$$
\begin{aligned}
k_{\beta} & \left(\lambda_{1}+\frac{\varepsilon}{q}\right)\left(2 \csc ^{2} \alpha+o(1)\right) \\
& >k\left(2 \csc ^{2} \alpha+o(1)-\varepsilon O(1)\right)^{\frac{1}{p}}\left(2 \csc ^{2} \beta+\tilde{o}(1)\right)^{\frac{1}{q}},
\end{aligned}
$$

and then

$$
\frac{4 \pi^{2}}{\lambda^{2} \sin ^{2}\left(\frac{\pi \lambda_{1}}{\lambda}\right)} \csc ^{2} \beta \csc ^{2} \alpha \geq 2 k \csc ^{\frac{2}{p}} \alpha \csc ^{\frac{2}{q}} \beta \quad\left(\varepsilon \rightarrow 0^{+}\right)
$$

namely, $k\left(\lambda_{1}\right)=\frac{2 \pi^{2}}{\lambda^{2} \sin ^{2}\left(\frac{\pi \lambda_{1}}{\lambda}\right)} \csc ^{\frac{2}{p}} \beta \csc ^{\frac{2}{q}} \alpha \geq k$. Hence, $k=k\left(\lambda_{1}\right)$ is the best possible constant factor of (17).

The constant factor $k\left(\lambda_{1}\right)$ in (18) and (19) is still the best possible. Otherwise, we would reach a contradiction by (27) and (28) that the constant factor in (17) is not the best possible.

Remark 2 (i) For $\xi=\eta=0$ in (20), setting

$$
\tilde{\theta}_{1}\left(\lambda_{2}, m\right):=\left[\frac{\lambda}{\pi} \sin \left(\frac{\pi \lambda_{1}}{\lambda}\right)\right]^{2} \int_{0}^{\frac{\ln 2}{\ln |m|}} \frac{\ln u}{u^{\lambda}-1} u^{\lambda_{2}-1} d u=O\left(\frac{1}{\ln ^{\lambda_{2} / 2}|m|}\right) \in(0,1)
$$


we have the following new inequality:

$$
\begin{aligned}
\sum_{|n|=2}^{\infty} & \sum_{|m|=2}^{\infty} \frac{\ln (\ln |m| / \ln |n|)}{\ln ^{\lambda}|m|-\ln ^{\lambda}|n|} a_{m} b_{n} \\
> & \frac{2 \pi^{2}}{\left[\lambda \sin \left(\frac{\pi \lambda_{1}}{\lambda}\right)\right]^{2}} \\
& \times\left[\sum_{|m|=2}^{\infty}\left(1-\tilde{\theta}_{1}\left(\lambda_{2}, m\right)\right) \frac{\ln ^{p\left(1-\lambda_{1}\right)-1}|m|}{|m|^{1-p}} a_{m}^{p}\right]^{\frac{1}{p}}\left[\sum_{|n|=2}^{\infty} \frac{\ln ^{q\left(1-\lambda_{2}\right)-1}|n|}{|n|^{1-q}} b_{n}^{q}\right]^{\frac{1}{q}} .
\end{aligned}
$$

It follows that (20) is an extension of (29). In particular, for $\lambda=1, \lambda_{1}=\lambda_{2}=\frac{1}{2}$, setting

$$
\tilde{\theta}_{1}(m):=\frac{1}{\pi^{2}} \int_{0}^{\frac{\ln 2}{\ln |m|}} \frac{\ln u}{u-1} u^{\frac{-1}{2}} d u=O\left(\frac{1}{\ln ^{1 / 4}|m|}\right) \in(0,1)
$$

we have the following simple reverse Mulholland-type inequality in the whole plane:

$$
\begin{aligned}
& \sum_{|n|=2}^{\infty} \sum_{|m|=2}^{\infty} \frac{\ln (\ln |m| / \ln |n|)}{\ln (|m| /|n|)} a_{m} b_{n} \\
& \quad>2 \pi^{2}\left[\sum_{|m|=2}^{\infty}\left(1-\tilde{\theta}_{1}(m)\right) \frac{\ln \frac{p}{2}-1|m|}{|m|^{1-p}} a_{m}^{p}\right]^{\frac{1}{p}}\left(\sum_{|n|=2}^{\infty} \frac{\ln \frac{q}{2}-1|n|}{|n|^{1-q}} b_{n}^{q}\right)^{\frac{1}{q}} .
\end{aligned}
$$

(ii) If $a_{-m}=a_{m}, b_{-n}=b_{n}(m, n \in \mathbf{N} \backslash\{1\})$, for $m \in \mathbf{N} \backslash\{1\}$, setting

$$
\begin{aligned}
& \widehat{\theta}_{1}\left(\lambda_{2}, m\right):=\left[\frac{\lambda}{\pi} \sin \left(\frac{\pi \lambda_{1}}{\lambda}\right)\right]^{2} \int_{0}^{\frac{\ln (2+\eta)}{\ln (m-\xi)}} \frac{\ln u}{u^{\lambda}-1} u^{\lambda_{2}-1} d u=O\left(\frac{1}{\ln ^{\lambda_{2} / 2}(m-\xi)}\right) \in(0,1), \\
& \breve{\theta}_{1}\left(\lambda_{2}, m\right):=\left[\frac{\lambda}{\pi} \sin \left(\frac{\pi \lambda_{1}}{\lambda}\right)\right]^{2} \int_{0}^{\ln (2+\eta)} \frac{\ln u}{\ln (m+\xi)} \frac{\ln }{u^{\lambda}-1} u^{\lambda_{2}-1} d u=O\left(\frac{1}{\ln ^{\lambda_{2} / 2}(m+\xi)}\right) \in(0,1),
\end{aligned}
$$

(20) reduces to

$$
\begin{aligned}
& \sum_{n=2}^{\infty} \sum_{m=2}^{\infty}\left\{\frac{\ln [\ln (m-\xi) / \ln (n-\eta)]}{\ln ^{\lambda}(m-\xi)-\ln ^{\lambda}(n-\eta)}+\frac{\ln [\ln (m-\xi) / \ln (n+\eta)]}{\ln ^{\lambda}(m-\xi)-\ln ^{\lambda}(n+\eta)}\right. \\
&\left.\quad+\frac{\ln [\ln (m+\xi) / \ln (n-\eta)]}{\ln ^{\lambda}(m+\xi)-\ln ^{\lambda}(n-\eta)}+\frac{\ln [\ln (m+\xi) / \ln (n+\eta)]}{\ln ^{\lambda}(m+\xi)-\ln ^{\lambda}(n+\eta)}\right\} a_{m} b_{n} \\
&> \frac{2 \pi^{2}}{\left[\lambda \sin \left(\frac{\pi \lambda_{1}}{\lambda}\right)\right]^{2}}\left\{\sum _ { m = 2 } ^ { \infty } \left[\left(1-\widehat{\theta}_{1}\left(\lambda_{2}, m\right)\right) \frac{\ln ^{p\left(1-\lambda_{1}\right)-1}(m-\xi)}{(m-\xi)^{1-p}}\right.\right. \\
&\left.\left.+\left(1-\breve{\theta}_{1}\left(\lambda_{2}, m\right)\right) \frac{\ln ^{p\left(1-\lambda_{1}\right)-1}(m+\xi)}{(m+\xi)^{1-p}}\right] a_{m}^{p}\right\}^{\frac{1}{p}} \\
& \times\left\{\sum_{n=2}^{\infty}\left[\frac{\ln ^{q\left(1-\lambda_{2}\right)-1}(n-\eta)}{(n-\eta)^{1-q}}+\frac{\ln ^{q\left(1-\lambda_{2}\right)-1}(n+\eta)}{(n+\eta)^{1-q}}\right] b_{n}^{q}\right\}^{\frac{1}{q}} .
\end{aligned}
$$


In particular, for $\xi=\eta=0, \lambda=1, \lambda_{1}=\lambda_{2}=\frac{1}{2}$, setting

$$
\widehat{\theta}_{1}(m):=\frac{1}{\pi^{2}} \int_{0}^{\frac{\ln 2}{\ln m}} \frac{\ln u}{u-1} u^{-\frac{1}{2}} d u=O\left(\frac{1}{\ln ^{1 / 4} m}\right) \in(0,1)
$$

we have the following simple reverse Mulholland-type inequality:

$$
\begin{aligned}
& \sum_{n=2}^{\infty} \sum_{m=2}^{\infty} \frac{\ln (\ln m / \ln n)}{\ln (m / n)} a_{m} b_{n} \\
& \quad>\pi^{2}\left[\sum_{m=2}^{\infty}\left(1-\widehat{\theta}_{1}(m)\right) \frac{\ln ^{\frac{p}{2}-1} m}{m^{1-p}} a_{m}^{p}\right]^{\frac{1}{p}}\left(\sum_{n=2}^{\infty} \frac{\ln ^{\frac{q}{2}-1} n}{n^{1-q}} b_{n}^{q}\right)^{\frac{1}{q}} .
\end{aligned}
$$

\section{Conclusions}

In this paper, we obtain a new reverse Mulholland's inequality in the whole plane with a best possible constant factor in Theorems 1-2. Equivalent forms and a few particular cases are considered. The method of real analysis is very important and is the key to prove the reverse equivalent inequalities with the best possible constant factor. The lemmas and theorems can provide an extensive account of this type inequalities.

\footnotetext{
Acknowledgements

This work is supported by the National Natural Science Foundation (Nos. 61370186, 61640222, and 11401113) and Science and Technology Planning Project Item of Guangzhou City (No. 201707010229).
}

\section{Competing interests}

The authors declare that they have no competing interests.

\section{Authors' contributions}

BY carried out the mathematical studies, participated in the sequence alignment, and drafted the manuscript. JL participated in the design of the study and performed the numerical analysis. Both authors read and approved the final manuscript.

\section{Publisher's Note}

Springer Nature remains neutral with regard to jurisdictional claims in published maps and institutional affiliations.

Received: 10 January 2018 Accepted: 2 April 2018 Published online: 10 April 2018

\section{References}

1. Hardy, G.H., Littlewood, J.E., Polya, G.: Inequalities. Cambridge University Press, Cambridge (1934)

2. Mitrinović, D.S., Pečarić, J.E., Fink, A.M.: Inequalities Involving Functions and Their Integrals and Derivatives. Kluwer Academic Publishers, Boston (1991)

3. Yang, B.: A new Hilbert's type integral inequality. Soochow J. Math. 33(4), 849-859 (2007)

4. Hong, Y.: All-sided generalization about Hardy-Hilbert integral inequalities. Acta Math. Sin. 44(4), 619-626 (2001)

5. Krnić, M., Pečarić, J.E.: General Hilbert's and Hardy's inequalities. Math. Inequal. Appl. 8(1), 29-51 (2005)

6. Perić, I., Vuković, P.: Multiple Hilbert's type inequalities with a homogeneous kernel. Banach J. Math. Anal. 5(2), 33-43 (2011)

7. He, B.: A multiple Hilbert-type discrete inequality with a new kernel and best possible constant factor. J. Math. Anal. Appl. 431, 902-990 (2015)

8. Adiyasuren, V., Tserendorj, B., Krnić, M.: Multiple Hilbert-type inequalities involving some differential operators. Banach J. Math. Anal. 10(2), 320-337 (2016)

9. Li, Y., He, B.: On inequalities of Hilbert's type. Bull. Aust. Math. Soc. 76(1), 1-13 (2007)

10. Krnić, M., Vuković, P.: On a multidimensional version of the Hilbert type inequality. Anal. Math. 38(4), 291-303 (2012)

11. Huang, Q., Yang, B.: A more accurate half-discrete Hilbert inequality with a nonhomogeneous kernel. J. Funct. Spaces Appl. 2013, Article ID 628250 (2013)

12. He, B., Wang, Q.: A multiple Hilbert-type discrete inequality with a new kernel and best possible constant factor. J. Math. Anal. Appl. 431(2), 889-902 (2015)

13. Yang, B., Chen, Q.: A new extension of Hardy-Hilbert's inequality in the whole plane. J. Funct. Spaces 2016, Article ID $9197476(2016)$

14. Xin, D., Yang, B., Chen, Q.: A discrete Hilbert-type inequality in the whole plane. J. Inequal. Appl. 2016, 133 (2016) 
15. Zhong, Y., Yang, B., Chen, Q.: A more accurate Mulholland-type inequality in the whole plane. J. Inequal. Appl., 2017 315 (2017)

16. Yang, B.: A more accurate multidimensional Hardy-Hilbert's inequality. J. Appl. Anal. Comput. 8(2), 559-573 (2018)

17. Kuang, J.: Applied Inequalities. Shangdong Science Technic Press, Jinan (2010) (in Chinese)

Submit your manuscript to a SpringerOpen ${ }^{\odot}$ journal and benefit from:

- Convenient online submission

- Rigorous peer review

- Open access: articles freely available online

- High visibility within the field

Retaining the copyright to your article

Submit your next manuscript at $\boldsymbol{\nabla}$ springeropen.com 